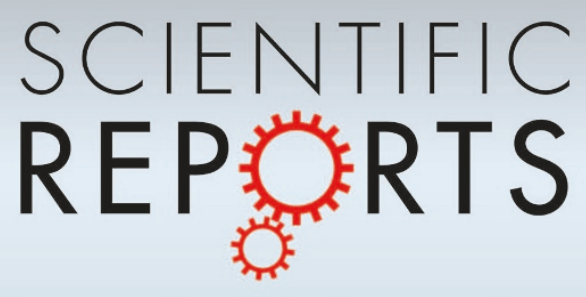

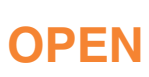

SUBJECT AREAS:

COMPUTER SCIENCE

COMPUTATIONAL SCIENCE

COMPLEX NETWORKS

Received

6 May 2014

Accepted

7 August 2014

Published

9 September 2014

Correspondence and requests for materials should be addressed to

Z.Z.Z. (zhangzz@ fudan.edu.cn)

\section{Controlling the efficiency of trapping in a scale-free small-world network}

\author{
Yuan Lin ${ }^{1,2} \&$ Zhongzhi Zhang ${ }^{1,2}$
}

'School of Computer Science, Fudan University, Shanghai 200433, China, ${ }^{2}$ Shanghai Key Lab of Intelligent Information Processing, Fudan University, Shanghai 200433, China.

Designing appropriate techniques to effectively control the trapping process in complex systems towards desirable efficiency is of paramount importance in the study of trapping problem. In this paper, we present three different methods guiding trapping process in a scale-free small-world network with a deep trap positioned at an initial node. All the proposed approaches dominate the trapping process by varying the transition probability of random walks. In the first two techniques, the transition probability is modified by an introduced weight parameter and a stochastic parameter, respectively. And the third scheme is a combination of the first two approaches, controlled by both parameters synchronously. For all the three control strategies, we derive both analytically and numerically the average trapping time (ATT) as the measure of the trapping efficiency, with the obtained explicit expressions being in good agreement with their corresponding exact numerical solutions. Our results indicate that the weight parameter changes simultaneously the dominating scaling of ATT and its prefactor. Different from the weight parameter, the stochastic parameter only modifies the prefactor, keeping the leading scaling unchanged. Finally, compared with the first two manners, the third strategy is a fine control, possessing the advantages of the first two ones. This work deepens the understanding of controlling trapping process in complex systems.

$\mathrm{T}$ rapping is a paradigmatic dynamical process, which can describe a variety of other dynamical processes occurring in diverse complex systems, e.g., lighting harvesting ${ }^{1-5}$, energy transport ${ }^{6,7}$ and target searching ${ }^{8,9}$. Trapping problem constitutes an integral primary problem of random walks, defined as a kind of random walks taking place in networks in the presence of a perfect trap, absorbing all particles that visit $\mathrm{it}^{10}$. A basic quantity relevant to the trapping problem is trapping time, also called mean first-passage time (MFPT) ${ }^{11-16}$. The MFPT from a node $i$ to the trap is the expected time taken by a particle leaving from $i$ to reach the trap for the first time. The average trapping time (ATT) is defined as the average of MFPTs over all starting nodes other than the trap. The ATT is a primary indicator of trapping, since it measures the efficiency of trapping process. Hitherto, evaluating ATT has received considerable attention for diverse networks ${ }^{17-29}$.

Most previous works focused on unraveling the effects of geometrical structure on trapping efficiency. In the research area of trapping, another central problem is to control the dynamical process. Recently, controllability of complex networks has become a topic of active pursuit and has attracted extensive attention ${ }^{30-32}$. Within the framework of controllability, several basic issues related to network control have been addressed, including optimization of controllability ${ }^{33}$, energy required in control $^{34}$, centrality control ${ }^{35}$, controlling edge dynamics ${ }^{36}$, bimodality in control process ${ }^{37}$, and capacity control ${ }^{38}$. In the context of trapping in complex systems, it is desirable to design control techniques steering the trapping process towards wanted trapping efficiency. Significant efforts have been devoted to this problem in several networks, such as one-dimensional systems $\mathrm{s}^{39}$, dendrimers ${ }^{3}$, and treelike fractals ${ }^{40}$. However, related control approaches for trapping process in scale-free smallworld networks remains less understood, in spite of the fact that this network family displays some prominent features of real systems $s^{41-43}$.

Although real-life networks are often random, the study of artificial networks with well-controlled properties is also of high interest ${ }^{44}$. In this paper, we focus on the trapping process in a hierarchical small-world scale-free network ${ }^{45}$, which has received much recent interest ${ }^{46-51}$. We propose three control strategies to steer the trapping process with a perfect trap fixed at an initial node. All the three methods control the trapping efficiency by modifying the local transition probability. In the first technique, a positive tunable weight parameter is introduced, which acts as a similar role of energetic funnel in dendrimers ${ }^{1,3}$. The second approach is based on delaydiffusion $^{52,53}$, where a stochastic parameter is introduced to change the transition probability. The third manner is a combination of the first two methods, which is controlled simultaneously by the weight and stochastic parameters. 
For all the three control strategies, we derive analytically the ATT and their leading scalings, which are consistent with the numerical results. We show that the weight parameter has a strong effect on both the leading scaling and the prefactor of the trapping efficiency, by changing which the ATT can scale as a superlinear, linear, or sublinear function of the system size. The stochastic parameter associated with the delayed diffusion can only modify the coefficient of the dominating scaling for ATT, without changing the leading behavior of trapping efficiency. Finally, since the third approach combines the advantages of the first two methods, it can finely control the trapping process. This work opens new avenues to effectively control trapping process towards a desirable case with ideal efficiency.

\section{Results}

Construction and properties of the network. We first introduce the construction and some relevant features of the small-world scale-free network, which is built in an iterative manner. Let $\mathbb{F}_{n}(n \geq 0)$ denote the network after $n$ iterations (generations). Then, the construction of $\mathbb{F}_{n}$ can be described as follows. Initially $(n=0), \mathbb{F}_{0}$ contains two nodes connected by an edge. For any $n>0, \mathbb{F}_{n}$ is obtained from $\mathbb{F}_{n-1}$ by performing the following operation. For each edge in $\mathbb{F}_{n-1}$, a new node is introduced and linked to both end nodes of the edge. Figure 1 shows the construction algorithm schematically.

The particular construction allows determining relevant properties of $\mathbb{F}_{n}$. The number of newly introduced nodes at generation $i$ $(i \geq 1)$ is $\bar{N}_{i}=3^{i-1}$. Thus, the number of nodes $N_{n}$ in $\mathbb{F}_{n}$ is

$$
N_{n}=2+\sum_{i=1}^{N} \bar{N}_{i}=\frac{1}{2}\left(3^{n}+3\right) \text {. }
$$

Let $\Omega_{n}$ represent the set of nodes in $\mathbb{F}_{n}$, and let $\bar{\Omega}_{n}$ be the set of nodes created at generation $n$. It is evident that $\Omega_{n}=\Omega_{n-1} \cup \bar{\Omega}_{n},\left|\Omega_{n}\right|=N_{n}$ $=\left(3^{n}+3\right) / 2$, and $\left|\bar{\Omega}_{n}\right|=\bar{N}_{n}=3^{n-1}$, where $|\Omega|$ denotes the number of elements in set $\Omega$.

In network $\mathbb{F}_{n}$, the degree of all nodes created at the same iteration is identical. Let $k_{i}(n)$ stand for the degree of node $i$ in $\mathbb{F}_{n}$, which fulfils the relation

$$
k_{i}(n+1)=2 k_{i}(n) .
$$

Let $K_{n}$ denote the sum of degrees for all nodes. Then,

$$
K_{n}=2 K_{n-1}+2 \bar{N}_{n}
$$
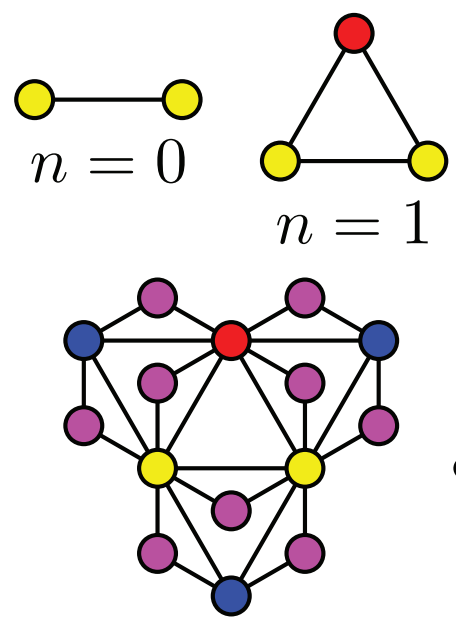

$$
n=3
$$

Figure 1 | Illustration for the evolution process of the small-world scalefree network. which, together with the initial condition $K_{0}=2$, leads to

$$
K_{n}=2 \times 3^{n} .
$$

Thus, the average degree of $\mathbb{F}_{n}$ is $\langle k\rangle_{n}=K_{n} / N_{n}=\left(4 \times 3^{n-1}\right) /\left(3^{n-1}+\right.$ $1)$, which is approximatively equal to 4 when the network is very large, indicating that the network is sparse.

The network being studied presents some remarkable properties as observed in most real systems ${ }^{45,47,48}$. It is scale-free with the degree of nodes following a power-law degree distribution $P(k) \sim k^{-\gamma}$, where the exponent $\gamma=1+\ln 3 / \ln 2$. Its average path length, defined as the mean of the shortest distance over all pairs of nodes, increases logarithmically with the network size $e^{45,49}$. Moreover, in the large network size limit, its average clustering coefficient tends to $4 / 5$. Thus, the network exhibits the small-world effect ${ }^{54}$.

Formulation of trapping in the network with a perfect trap at an initial hub node. As shown above, the goal of the present paper is to introduce three techniques to control the trapping process in the scale-free small-world network $\mathbb{F}_{n}$. Since any trapping process is dominated by the transition probability from one node to another, below we will address three control approaches by modifying the transition probability in different ways. In the first method, we change the transition probability by introducing a weight for every edge, which leads to qualitatively different behavior in the scaling of ATT; in the second approach, we modify the transition probability according to the birth times of different nodes, which cannot change the leading scaling of ATT but can vary its prefactor. The third manner is a combination of the former two approaches, which can alter the ATT both qualitatively and quantitatively.

Before introducing the control methods, we first formulate the problem of trapping in $\mathbb{F}_{n}$ in the presence of a deep trap located at an initial node. We focus on the discrete-time trapping problem described by discrete-time random walks. For the convenience of description, we label all the nodes in $\mathbb{F}_{n}$ in the following way. The initial two nodes in $\mathbb{F}_{0}$ are labeled as 1 and 2, respectively. In each new generation, only the new nodes created at this generation are labeled, while the labels of all old nodes remain unchanged, i.e., we label new nodes in $\mathbb{F}_{n}$ as $N_{n-1}+1, N_{n-1}+2, \cdots, N_{n}$. Without loss of generality, we assume that the trap is placed at the initial node labeled by 1. Note that throughout the whole paper, we only consider the nearest-neighbor random walks, where the walker is not allowed to stay put. At each time step, the probability of the walker moving from its current position, say node $i(i \geq 2)$, to one of its neighboring nodes $j(j$ $\neq i)$ is $p_{i j}$, satisfying $\sum_{j=1}^{N_{n}} p_{i j}=1$. Moreover, for two different adjacent nodes $j$ and $k$ of node $i$, the transition probability $p_{i j}$ might be not equal to $p_{i k}$.

Let $T_{i}^{(n)}$ stand for the trapping time, that is, the expected time for a walker starting from node $i$ to arrive at the trap in $\mathbb{F}_{n}$ for the first time. By definition, $T_{i}^{(n)}$ obeys the following relation

$$
T_{i}^{(n)}=\sum_{j=2}^{N_{n}} p_{i j} T_{j}^{(n)}+1 .
$$

Equation (5) characterizes the Markovian property of the random walks, which can be recast in matrix notation as

$$
\mathbf{T}=\mathbf{P T}+\mathbf{e}
$$

where $\mathbf{P}=\left[p_{i j}\right]$ is a matrix of order $N_{n}-1$, $\mathbf{T}=\left(T_{2}^{(n)}, T_{3}^{(n)}, \cdots T_{N_{n}}^{(n)}\right)^{\top}$ is an $\left(N_{n}-1\right)$-dimensional vector, and $\mathbf{e}$ is the $\left(N_{n}-1\right)$-dimensional vector of ones, i.e., $\mathbf{e}=(\mathbf{1}, \mathbf{1}, \cdots, \mathbf{1})^{\top}$. From equation (6), we obtain 


$$
\mathbf{T}=(\mathbf{I}-\mathbf{P})^{-1} \mathbf{e},
$$

where $\mathrm{I}$ is the $\left(N_{n}-1\right) \times\left(N_{n}-1\right)$ identity matrix.

Using equation (7), the ATT, denoted by $\langle T\rangle_{n}$, which is the mean of $T_{i}^{(n)}$ over all non-trap starting nodes distributed uniformly over the whole network $\mathbb{F}_{n}$, can be obtained by

$$
\langle T\rangle_{n}=\frac{1}{N_{n}-1} \sum_{i=2}^{N_{n}} T_{i}^{(n)}=\frac{1}{N_{n}-1} \sum_{i=2}^{N_{n}} \sum_{j=2}^{N_{n}} \tau_{i j},
$$

where $\tau_{i j}$ is the $i j$ th element of matrix $(\mathbf{I}-\mathbf{P})^{-1}$. The quantity $\langle T\rangle_{n}$ is significantly important since it is a quantitative indicator measuring the trapping efficiency: the less the ATT, the more efficient the trapping process, and vice versa.

Equation (8) shows that the problem of determining ATT $\langle T\rangle_{n}$ can be reduced to finding the sum of all elements of a matrix. Although the computation of inverting a matrix puts heavy demands on time and memory for large networks, equation (8) can be used to check the results for ATT derived by other methods, at least for networks with a small number of nodes. In what follows, we will apply equation (8) to verify our analytical solutions for the three proposed control techniques.

Qualitatively controlling the trapping efficiency by introducing edge weight. The first control method changes the local transition probability through introducing a weight for each edge. As will be shown, using this method, the ATT can behave superlinealy, linearly, or sublinearly, with the system size.

Numerical solution to ATT in a weighted scale-free small-world network. The aforementioned binary network $\mathbb{F}_{n}$ can be extended to a weighted network, by introducing a weight parameter $w(w>0)$ in the construction algorithm. We use $\mathbb{W}_{n}$ to denote the weighted network, which is built as follows. For $n=0, \mathbb{W}_{0}$ is composed of two nodes linked by an edge with unit weight. For $n \geq 1, \mathbb{W}_{n}$ is derived from $\mathbb{W}_{n-1}$ in the following way. For each existing edge in $\mathbb{W}_{n-1}$, a new node is created and attached to both end nodes of the edge by two new links. The weight of both new edges is $w$ times that of the original edge giving birth to the two new edges. Repeating the iterative steps, we obtain the weighted version of $\mathbb{F}_{n}$. Note that $\mathbb{W}_{n}$ reduces to the binary case $\mathbb{F}_{n}$ when $w=1$.

By construction, the total strength $S_{n}$ of network $\mathbb{W}_{n}$, namely the sum of weights of all edges, follows the recursive relation:

$$
S_{n}=S_{n-1}+2 w S_{n-1} \text {. }
$$

Considering $S_{0}=1$, we have

$$
S_{n}=(2 w+1)^{n} .
$$

Mathematically, the properties of network $\mathbb{W}_{n}$ are reflected in the generalized adjacency matrix (weight matrix) $\mathrm{W}$ with the element $w_{i j}$ specifying the weight of the edge linking nodes $i$ and $j$. Let $s_{i}$ denote the strength ${ }^{55}$ of node $i$ in $\mathbb{W}_{n}$, which is the sum of weights over all edges incident to $i$. In other words, $s_{i}=\sum_{j=1}^{N_{n}} w_{i j}$. Notice that $s_{i}$ is the $i$ th nonzero entry of the diagonal strength matrix $\mathbf{S}=\operatorname{diag}\left(s_{1}, s_{2}, \cdots, s_{N_{n}}\right)$ of network $\mathbb{W}_{n}$.

After defining the weighted network $\mathbb{W}_{n}$, we now study trapping in $\mathbb{W}_{n}$ with a deep trap positioned at an initial node 1 . For discretetime random walks in $\mathbb{W}_{n}$, at each time step, the walker moves from its current location, say node $i$, to one of its neighboring nodes $j$ by probability $p_{i j}=w_{i j} / s_{i}$. In fact, all the entries $p_{i j}\left(2 \leq i, j \leq N_{n}\right)$ constitute matrix $\mathbf{P}$ that is an $\left(N_{n}-1\right) \times\left(N_{n}-1\right)$ sub-matrix of $\mathrm{S}^{-1} \mathrm{~W}$ with the row and column corresponding to trap being removed. Thus, the ATT $\langle T\rangle_{n}$ can be computed by using equation (8).
In addition to equation (8), the exact value of ATT $\langle T\rangle_{n}$ for trapping in $\mathbb{W}_{n}$ can be also determined in terms of the eigenvalues and eigenvectors of the Laplacian matrix of network $\mathbb{W}_{n}$, by applying the universal framework for random walks in weighted networks ${ }^{56}$. We use $\mathrm{L}$ to represent the Laplacian matrix of $\mathbb{W}_{n}$, defined as $\mathrm{L}=\mathrm{S}-\mathbf{W}$. Let $\lambda_{1}, \lambda_{2}, \cdots, \lambda_{N_{n}}$ be the $N_{n}$ eigenvalues of $\mathrm{L}$, rearranged as $0=\lambda_{1}<\lambda_{2}<\cdots<\lambda_{N_{n}}$, and let $\mu_{1}, \mu_{2}, \cdots, \mu_{N_{n}}$ be their corresponding mutually orthogonal eigenvectors of unit length, where $\mu_{i}=\left(\mu_{i 1}, \mu_{i 2}, \cdots, \mu_{i N_{n}}\right)^{\top}$. Then, the quantity $\langle T\rangle_{n}$ can be represented as

$$
\langle T\rangle_{n}=\frac{N_{n}}{N_{n}-1} \sum_{k=2}^{N_{n}} \frac{1}{\lambda_{k}}\left(S_{n} \times \mu_{k 1}^{2}-\mu_{k 1} \sum_{z=1}^{N_{n}} s_{z} \mu_{k z}\right) .
$$

We have performed extensive numerical computation for $\langle T\rangle_{n}$ using both equations (8) and (11), the results of which are consistent with each other. In the following text, we will utilize these numerical results to check our analytical expression for $\langle T\rangle_{n}$.

Although one can employ equations (8) or (11) to obtain numerically but exactly the ATT $\langle T\rangle_{n}$, either inverting a matrix or determining the full spectrum of a matrix is prohibitively time and memory consuming, making it intractable to employ equations (8) or (11) to compute $\langle T\rangle_{n}$ for a large network. In particular, by making use of equations (8) or (11), it is hard and even impossible to obtain asymptotic scaling for the ATT $\langle T\rangle_{n}$. Hence, it is of utmost importance to develop a computationally cheaper method for computing $\langle T\rangle_{n}$. Fortunately, the peculiar construction and structure of the studied network allow to analytically treat $\langle T\rangle_{n}$ as well as the ATT for the other two trapping problems, obtaining closed-form solutions.

Analytical solution to ATT. For the purpose of studying ATT analytically, let's begin with examining random walks in $\mathbb{W}_{n+1}$. By construction, upon growth of the network from generation $n$ to next generation $n+1$, the degree of node $i$ doubles, that is, it grows from $k_{i}(n)$ to $k_{i}(n+1)=2 k_{i}(n)$. These $2 k_{i}(n)$ neighbors of $i$ can be classified into two categories: one half are the original neighbors belonging to $\Omega_{n}$, and the other half are newly introduced neighbors in $\bar{\Omega}_{n+1}$. Let $Z$ represent the MFPT for going from node $i$ to any of its $k_{i}(n)$ old neighbors; and let $X$ be the MFPT for going from any of the $k_{i}(n)$ new neighbors of $i$ to one of its $k_{i}(n)$ old neighbors. Then we can establish the following underlying backward equations:

$$
\left\{\begin{array}{c}
Z=\frac{1}{1+w}+\frac{w}{1+w}(1+X), \\
X=\frac{1}{2}+\frac{1}{2}(1+Z),
\end{array}\right.
$$

which lead to $Z=(4 w+2) /(w+2)$. Thus, upon the evolution of the weighted network from generation $n$ to generation $n+1$, the trapping time for an arbitrary node $i$ increases by a factor of $(4 w+2) /(w$ +2 ), that is,

$$
T_{i}^{(n+1)}=\frac{4 w+2}{w+2} T_{i}^{(n)}
$$

which will be helpful for the following evaluation of the exact formula for $\langle T\rangle_{n}$.

Having obtained the evolution rule of trapping time for any node in the weighted network, we continue to determine the ATT in $\mathbb{W}_{n}$. For this purpose, we define the following intermediary quantities for $1 \leq g \leq n$ :

$$
T_{g, \mathrm{tot}}^{(n)}=\sum_{i \in \Omega_{g}} T_{i}^{(n)}
$$

and

$$
\bar{T}_{g, \text { tot }}^{(n)}=\sum_{i \in \bar{\Omega}_{g}} T_{i}^{(n)}
$$


Then, $\langle T\rangle_{n}$ is given by

$$
\langle T\rangle_{n}=\frac{1}{N_{n}-1} T_{n, \text { tot }}^{(n)}
$$

which reduces the problem of evaluating $\langle T\rangle_{n}$ to determining $T_{n \text {,tot }}^{(n)}$.

By definition,

$$
T_{n, \text { tot }}^{(n)}=T_{n-1, \text { tot }}^{(n)}+\bar{T}_{n, \text { tot }}^{(n)}=\frac{4 w+2}{w+2} T_{n-1, \text { tot }}^{(n-1)}+\bar{T}_{n, \text { tot }}^{(n)},
$$

where equation (13) is used. Equation (17) indicates that, in order to obtain $T_{n \text {,tot }}^{(n)}$, we should first deduce $\bar{T}_{n \text {,tot }}^{(n)}$.

By construction, for each new node $x$ in $\bar{\Omega}_{n}$, it has only two neighbors $u$ and $v$, both belonging to $\Omega_{n-1}$. The ATTs of these three nodes obey

$$
T_{x}^{(n)}=1+\frac{1}{2} T_{u}^{(n)}+\frac{1}{2} T_{v}^{(n)} .
$$

Summing equation (18) over all nodes in $\bar{\Omega}_{n}$, we obtain

$$
\bar{T}_{n, \text { tot }}^{(n)}=\left|\bar{\Omega}_{n}\right|+\sum_{i \in \Omega_{n-1}}\left[k_{i}(n-1) \times \frac{1}{2} T_{i}^{(n)}\right],
$$

based on which we can further derive

$$
\bar{T}_{n+1, \text { tot }}^{(n+1)}=\left|\bar{\Omega}_{n+1}\right|+\sum_{i \in \Omega_{n}}\left[k_{i}(n) \times \frac{1}{2} T_{i}^{(n+1)}\right]
$$

Equation (20) minus equation (19) times $2(4 w+2) /(w+2)$ and recalling equations (2) and (13), we obtain the following recursive relation:

$$
\begin{aligned}
& \bar{T}_{n+1, \text { tot }}^{(n+1)}-\frac{8 w+4}{w+2} \bar{T}_{n, \text { tot }}^{(n)} \\
& =\left|\bar{\Omega}_{n+1}\right|-\frac{8 w+4}{w+2}\left|\bar{\Omega}_{n}\right|+\sum_{i \in \bar{\Omega}_{n}} T_{i}^{(n+1)} \\
& =\left|\bar{\Omega}_{n+1}\right|-\frac{8 w+4}{w+2}\left|\bar{\Omega}_{n}\right|+\frac{4 w+2}{w+2} \bar{T}_{n, \text { tot }}^{(n)}
\end{aligned}
$$

Using $\left|\bar{\Omega}_{n}\right|=3^{n-1}$ and $\bar{T}_{1, \text { tot }}^{(1)}=3(w+1) /(w+2)$, equation (21) can be resolved inductively to yield

$$
\bar{T}_{n, \mathrm{tot}}^{(n)}=\frac{3^{n-3}}{2 w}\left[(11 w+4)\left(4-\frac{6}{w+2}\right)^{n}+10 w-4\right] .
$$

Substituting equation (22) into equation (17) leads to

$$
\begin{aligned}
T_{n, \text { tot }}^{(n)}= & \frac{4 w+2}{w+2} T_{n-1, \text { tot }}^{(n-1)}+ \\
& \frac{3^{n-3}}{2 w}\left[(11 w+4)\left(4-\frac{6}{w+2}\right)^{n}+10 w-4\right] .
\end{aligned}
$$

Considering $T_{1, \text { tot }}^{(1)}=1+3(2 w+1) /(w+2)$, equation (23) is solved to yield

$$
T_{n, \mathrm{tot}}^{(n)}=\frac{3^{n-1}}{2}\left(2 \times 3^{n}+n+4\right)
$$

and

$$
\begin{aligned}
T_{n, \text { tot }}^{(n)} & =\frac{1}{36 w(w-4)}\left[(11 w+4)(w-4)\left(\frac{6+12 w}{w+2}\right)^{n}\right. \\
& \left.+\left(45 w^{2}-72 w\right)\left(\frac{4 w+2}{w+2}\right)^{n}+3^{n}\left(16-32 w-20 w^{2}\right)\right]
\end{aligned}
$$

for $w=4$ and $w \neq 4$, respectively.

Plugging the results in equations (24) and (25) into equation (16), we arrive at the closed-from expressions for $\langle T\rangle_{n}$ given by

$$
\langle T\rangle_{n}=\frac{2 \times 3^{n}+n+4}{3^{n}+3} \times 3^{n-1}
$$

and

$$
\begin{aligned}
\langle T\rangle_{n} & =\frac{1}{18 w(w-4)\left(3^{n}+3\right)}\left[(11 w+4)(w-4) 3^{n}\left(\frac{4 w+2}{w+2}\right)^{n}\right. \\
& \left.+\left(45 w^{2}-72 w\right)\left(\frac{4 w+2}{w+2}\right)^{n}+3^{n}\left(16-32 w-20 w^{2}\right)\right]
\end{aligned}
$$

for $w=4$ and $w \neq 4$, respectively. When $w=1, \mathbb{W}_{n}$ reduces to the unweighted network $\mathbb{F}_{n}$, and equation (27) is consistent with the result previously obtained in Ref. 57 .

We have checked our analytical formulas against numerical results obtained from equations (8) and (11). For different values of $w$ and $n$, the analytical results obtained from equations (26) and (27) completely agree with those numerical ones; see Fig. 2. This agreement serves as an independent test of our theoretical formulas.

Equations (26) and (27) show that for trapping in $\mathbb{W}_{n}$, the exact expression of ATT to an initial hub node is dependent on the weight parameter $w$. We now proceed to show how the leading behavior of ATT scales with the number of nodes in network $\mathbb{W}_{n}$.

Leading scalings of ATT. Recalling $N_{n}=\left(3^{n}+3\right) / 2$, equation (26) shows that $\langle T\rangle_{n} \sim N_{n}$ for $w=4$. When $w \neq 4$, the first term on the right-hand side (rhs) of equation (27) dominates, implying that $\langle T\rangle_{n} \sim N_{n}^{\log _{3}(4 w+2) /(w+2)}$. Therefore, in the whole range of $w>0$, the leading scaling of $\langle T\rangle_{n}$ can be unified as

$$
\langle T\rangle_{n} \sim N_{n}^{\eta(w)}=N_{n}^{\log _{3} \frac{4 w+2}{w+2}}
$$

where the exponent $\eta(w)=\log _{3} \frac{4 w+2}{w+2}$ is an increasing function of parameter $w$ and can be less than, equal to, or greater than 1 . Concretely, if $0<w<4, \eta(w)<1$, implying that $\langle T\rangle_{n}$ scales sublinearly with $N_{n}$; if $w=4, \eta(w)=1$, implying that $\langle T\rangle_{n}$ behaves linearly with $N_{n}$; and if $w>4, \eta(w)>1$, implying that $\langle T\rangle_{n}$ varies superlinearly with $N_{n}$.

Equation (28) indicates that the parameter $w$ has a significant impact on the trapping efficiency, by adjusting which the ATT can display rich behavior revealed by exponent $\eta(w)$. When $w$ grows from zero to infinite, $\eta(w)$ increases monotonously from 0 to $\log _{3} 4$. Thus, one can adjust the parameter $w$ to modify the transition probability and qualitatively control the trapping process.

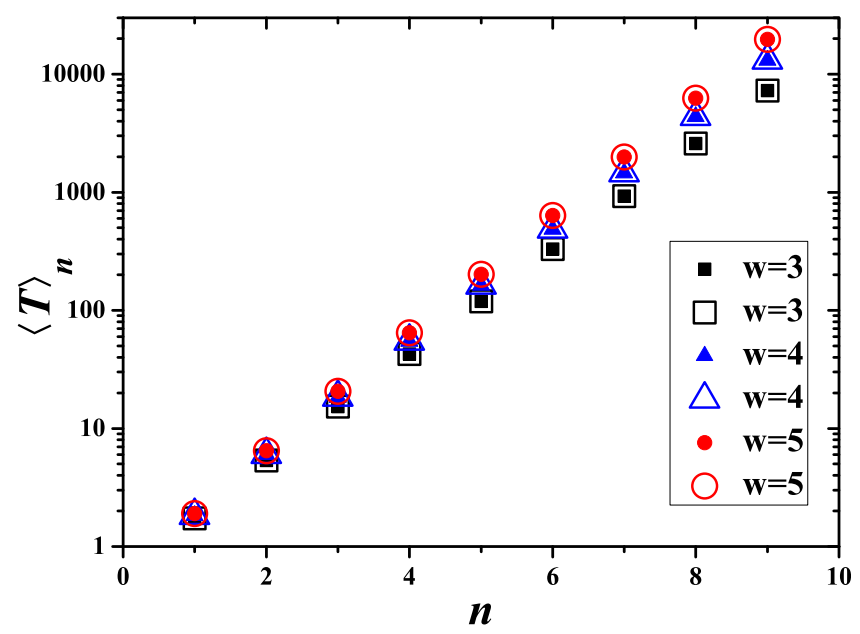

Figure $2 \mid\langle T\rangle_{n}$ as a function of $\boldsymbol{n}$ for three different values of $\boldsymbol{w}$. The filled symbols are the data coming from numerical results obtained by direct calculation from equations (8) and (11); while the empty symbols correspond to the exact analytical values given by equations (26) or (27). 
Quantitatively modifying the trapping efficiency by delayed random walks. We now present a control technique to quantitatively alter the trapping efficiency. It is often the case, when a network assembly is finished, its new topology does not come into play in dynamical processes right now. That is, there is delay for the new structure to influence dynamical processes taking place on the network. This phenomenon inspires us to propose the second control strategy that includes the delay of operation for new structure.

Definition of delayed random walks. By construction, the structure of $\mathbb{F}_{n-1}$ is embedded in $\mathbb{F}_{n}$. Then, we can define the following random walks with delay, called delayed random walks, performed on the binary network $\mathbb{F}_{n}$. During the process of delayed random walks, a walker starting from an old node in $\mathbb{F}_{n}$, which is already existent in $\mathbb{F}_{n-1}$, is allowed to perform isotropic nearest-neighbor random walks in either $\mathbb{F}_{n-1}$ or $\mathbb{F}_{n}$, with respective probabilities $p$ and $1-p$ $(0 \leq p \leq 1)$. That is to say, for delayed random walks in $\mathbb{F}_{n}$, the transition probability from an old node $i$ to one of its adjacency nodes $j$ is given by

$$
p_{i j}=\left\{\begin{array}{c}
p \times \frac{1}{k_{i}(n-1)}+(1-p) \times \frac{1}{k_{i}(n)}, \quad j \in \Omega_{n-1}, \\
(1-p) \times \frac{1}{k_{i}(n)}, \quad j \in \bar{\Omega}_{n} .
\end{array}\right.
$$

If the current state of the walker is at a new node, then it performs uniform nearest-neighbor random walks in $\mathbb{F}_{n}$.

There are two limiting cases for the above-defined delayed random walks in $\mathbb{F}_{n}$. When $p=0$, it reduces to unbiased random walks in $\mathbb{F}_{n}$; when $p=1$, it is exactly unbiased random walks in $\mathbb{F}_{n-1}$. As expected, the probability parameter $p$ governs the process of delayed random walks in $\mathbb{F}_{n}$. Below we will show that by varying $p$, significant modification occurs in the prefactor of ATT, with its dominating behavior unchanged.

Closed-form expression for ATT. We here concentrate on delayed random walks in $\mathbb{F}_{n}$ with a perfect trap at an initial node. In this case, we use $F_{i}^{(n)}$ to represent the trapping time for a walker starting from node $i$ to first arrive at the trap in $\mathbb{F}_{n}$, and use $\langle F\rangle_{n}$ to denote the ATT. In order to determine $\langle F\rangle_{n}$, we define two quantities for $g \leq n$ : $F_{g, \text { tot }}^{(n)}=\sum_{i \in \Omega_{g}} F_{i}^{(n)}$ and $\bar{F}_{g \text {,tot }}^{(n)}=\sum_{i \in \bar{\Omega}_{g}} F_{i}^{(n)}$. Then, the ATT $\langle F\rangle_{n}$ is given by

$$
\langle F\rangle_{n}=\frac{1}{N_{n}-1} F_{n, \text { tot }}^{(n)}
$$

Next, we determine $F_{n \text {,tot }}^{(n)}$.

Before evaluating $F_{n \text {,tot }}^{(n)}$, we first derive the evolution rule of $F_{i}^{(n)}$. Analogous to random walks in $\mathbb{W}_{n+1}$, the quantities $Z$ and $X$ for delayed random walks follow relations:

$$
\left\{\begin{array}{l}
Z=p+(1-p)\left[\frac{1}{2}+\frac{1}{2}(1+X)\right] \\
X=\frac{1}{2}+\frac{1}{2}(1+Z)
\end{array}\right.
$$

which lead to $Z=2(3-p) /(3+p)$. Thus, we have

$$
F_{i}^{(n+1)}=\frac{2(3-p)}{3+p} T_{i}^{(n)}
$$

where $T_{i}^{(n)}$ is trapping time of node $i$ for random walks in $\mathbb{W}_{n}$, corresponding to the particular case of $w=1$.
Using equation (32), $F_{n \text {,tot }}^{(n)}$ can be computed as

$$
F_{n, \text { tot }}^{(n)}=F_{n-1, \text { tot }}^{(n)}+\bar{F}_{n, \text { tot }}^{(n)}=\frac{2(3-p)}{3+p} T_{n-1, \text { tot }}^{(n-1)}+\bar{F}_{n, \text { tot }}^{(n)} .
$$

Substituting $w=1$ into equation (25) gives $T_{n-1, \text { tot }}^{(n-1)}$ in equation (33):

$$
T_{n-1, \text { tot }}^{(n-1)}=\frac{1}{12}\left(5 \times 6^{n-1}+3 \times 2^{n-1}+4 \times 3^{n-1}\right) .
$$

Thus, to obtain $F_{n \text {,tot }}^{(n)}$, we only need to determine $\bar{F}_{n \text {,tot }}^{(n)}$.

By using a similar process as that for random walks in $\mathbb{W}_{n}$, we can obtain

$$
\begin{aligned}
\bar{F}_{n, \text { tot }}^{(n)} & =\left|\bar{\Omega}_{n}\right|+\sum_{i \in \Omega_{n-1}}\left[k_{i}(n-1) \times \frac{1}{2} F_{i}^{(n)}\right] \\
& =\left|\bar{\Omega}_{n}\right|+\sum_{i \in \Omega_{n-1}}\left[k_{i}(n-1) \times \frac{3-p}{3+p} T_{i}^{(n-1)}\right]
\end{aligned}
$$

and

$$
\bar{F}_{n+1, \text { tot }}^{(n+1)}=\left|\bar{\Omega}_{n+1}\right|+\sum_{i \in \Omega_{n}}\left[k_{i}(n) \times \frac{3-p}{3+p} T_{i}^{(n)}\right] .
$$

Equation (36) minus equation (35) times 4 and considering the relations $T_{i}^{(n)}=2 T_{i}^{(n-1)}$ and $k_{i}(n)=2 k_{i}(n-1)$, we obtain the recursive relation:

$$
\begin{aligned}
\bar{F}_{n+1, \text { tot }}^{(n+1)}-4 \bar{F}_{n, \text { tot }}^{(n)} & =\left|\bar{\Omega}_{n+1}\right|-4\left|\bar{\Omega}_{n}\right|+\frac{2(3-p)}{3+p} \sum_{i \in \bar{\Omega}_{n}} T_{i}^{(n)} \\
& =\left|\bar{\Omega}_{n+1}\right|-4\left|\bar{\Omega}_{n}\right|+\frac{2(3-p)}{3+p} \bar{T}_{n, \text { tot }}^{(n)} .
\end{aligned}
$$

Combining equation (23) for $w=1,\left|\bar{\Omega}_{n}\right|=3^{n-1}$ and the initial condition $\bar{F}_{1 \text {,tot }}^{(1)}=6 /(p+3)$, we can resolve equation (37) to obtain

$$
\bar{F}_{n, \text { tot }}^{(n)}=\frac{3^{n-2}}{2(3+p)}\left[5(3-p) \times 2^{n}+10 p+6\right] .
$$

Plugging equations (34) and (38) into equation (33) leads to the explicit expression for $F_{n \text {,tot }}^{(n)}$ :

$$
F_{n, \text { tot }}^{(n)}=\frac{3-p}{3+p} \times 2^{n-2}+\frac{5(3-p)}{2(3+p)} 6^{n-1}+3^{n-1},
$$

which, together with equation (30), produces the exact formula for the ATT $\langle F\rangle_{n}$ given by

$$
\langle F\rangle_{n}=\frac{1}{6\left(3^{n}+3\right)}\left[\frac{3-p}{3+p} \times 2^{n-2}+\frac{5(3-p)}{2(3+p)} 6^{n-1}+3^{n-1}\right] .
$$

We have confirmed the analytical solution in equation (40) by comparing it with equation (8). The analytical and numerical results are completely consistent with each other, indicating that the predicted formula in equation (40) is valid. In addition, for the two special cases of $p=0$ and $p=1$, equation (40) agrees with the values of $\langle T\rangle_{n}$ and $\langle T\rangle_{n-1}$ for random walks in $\mathbb{W}_{n}$ corresponding to $w=1$, also indicating the validity of equation (40).

Leading behavior of ATT and its prefactor. Considering $N_{n}=\left(3^{n}+3\right) / 2$, the expression for $\langle F\rangle_{n}$ in equation (40) can be represented in terms of the system size $N_{n}$ in the following form:

$$
\begin{aligned}
\langle F\rangle_{n} & =\frac{1}{12 N_{n}}\left[\frac{5(3-p)}{4(3+p)}\left(2 N_{n}-3\right)^{1+\log _{3} 2}\right. \\
& \left.+\frac{3-p}{4(3+p)}\left(2 N_{n}-3\right)^{\log _{3} 2}+\frac{2}{3} N_{n}-1\right],
\end{aligned}
$$


which provides the exact dependence of $\langle F\rangle_{n}$ on the network size $N_{n}$ and parameter $p$. For a large system, i.e., $N_{n} \rightarrow \infty$, we have the following expression for the dominating term of $\langle F\rangle_{n}$ :

$$
\langle F\rangle_{n} \approx \frac{5(3-p)}{24(3+p)}\left(2 N_{n}\right)^{\log _{3} 2} \sim N_{n}^{\log _{3} 2} .
$$

Equation (42) indicates that in the whole range of $0 \leq p \leq 1$, the ATT increases sublinearly with the system size $N_{n}$, with the exponent $\log _{3} 2$ independent of parameter $p$. Although parameter $p$ has little influence on the dominating behavior of ATT, it modifies significantly the prefactor $[5(3-p)] /[24(3+p)]$ of the dominating term of ATT, which is a descending function of $p$. When $p$ grows from 0 to 1 , the prefactor drops from $5 / 24$ to $5 / 48$, suggesting that the inclusion of delayed random walks can improve the trapping efficiency in a significant way. For the two extreme cases of $p=1$ and $p=0$, the ATT for the former with maximal delay is only one half of that for the latter without any delay.

Unifying control technique. Thus far, we have described two different approaches to control the efficiency for trapping in the network concerned. The first method can change the leading term of ATT and its coefficient simultaneously, while the second technique only modifies the prefactor but keeps the leading term unchanged. In the sequel, we will present a unifying control scheme incorporating the two previous methods.

Explicit formula for ATT. The new technique is in fact a delayed random walk in the weighted network $\mathbb{W}_{n}$. For this case of random walks, if the walker is currently at an old node belonging to $\Omega_{n-1}$, it can perform random walks either in $\mathbb{W}_{n-1}$ with probability $p$ or in $\mathbb{W}_{n}$ with complementary probability $1-p$; if the walker is currently at a new node belonging to $\bar{\Omega}_{n}$, it performs random walks in $\mathbb{W}_{n}$. Then the quantities $Z$ and $X$ obey the relations

$$
\left\{\begin{array}{lc}
Z & =p+(1-p)\left[\frac{1}{1+w}+\frac{w}{1+w}(1+X)\right], \\
X= & \frac{1}{2}+\frac{1}{2}(1+Z),
\end{array}\right.
$$

which give $Z=2(1+2 w-w p) /(2+w+w p)$.

For delayed random walks in $\mathbb{W}_{n}$ with a trap fixed at an initial node, we use $P_{i}^{(n)}$ to denote the trapping time for node $i$, and use $\langle P\rangle_{n}$ to represent the ATT to the trap. Then, from equation (43), we have

$$
P_{i}^{(n+1)}=\frac{2(1+2 w-w p)}{2+w+w p} T_{i}^{(n)} .
$$

In order to determine $\langle P\rangle_{n}$, for $g \leq n$, we define $P_{g \text {,tot }}=\sum_{i \in \Omega_{g}} P_{i}^{(n)}$ and $\bar{P}_{g \text {,tot }}^{(n)}=\sum_{i \in \bar{\Omega}_{g}} P_{i}^{(n)}$ as before. Then,

$$
\langle P\rangle_{n}=\frac{1}{N_{n}-1} P_{n, \text { tot }}^{(n)},
$$

where $P_{n, \text { tot }}^{(n)}$ can be rewritten as

$$
\begin{aligned}
P_{n, \text { tot }}^{(n)} & =P_{n-1, \text { tot }}^{(n)}+\bar{P}_{n, \text { tot }}^{(n)} \\
& =\frac{2(1+2 w-w p)}{2+w+w p} T_{n-1, \text { tot }}^{(n-1)}+\bar{P}_{n, \text { tot }}^{(n)} .
\end{aligned}
$$

The quantity $\bar{P}_{n \text {,tot }}^{(n)}$ can be evaluated as follows. First, it is easy to derive

$$
\bar{P}_{n, \text { tot }}^{(n)}=\left|\bar{\Omega}_{n}\right|+\sum_{i \in \Omega_{n-1}}\left[k_{i}(n-1) \times \frac{1+2 w-w p}{2+w+w p} T_{i}^{(n-1)}\right]
$$

and

$$
\bar{P}_{n+1, \text { tot }}^{(n+1)}=\left|\bar{\Omega}_{n+1}\right|+\sum_{i \in \Omega_{n}}\left[k_{i}(n) \times \frac{1+2 w-w p}{2+w+w p} T_{i}^{(n)}\right],
$$

both of which yield the relation

$$
\begin{aligned}
\bar{P}_{n+1, \text { tot }}^{(n+1)}= & \frac{2(4 w+2)}{w+2} \bar{P}_{n, \text { tot }}^{(n)}+\left|\Omega_{n+1}\right|-\frac{2(4 w+2)}{w+2}\left|\Omega_{n}\right| \\
& +\frac{2(1+2 w-w p)}{2+w+w p} \bar{T}_{n, \text { tot }}^{(n)} .
\end{aligned}
$$

Using $\bar{P}_{1 \text {,tot }}^{(1)}=(3+3 w) /(2+w+p w)$, equation (49) is solved to obtain

$$
\begin{aligned}
\bar{P}_{n, \text { tot }}^{(n)}= & \frac{3^{n-3}}{2 w(2 w+1)[(p+1) w+2]} \\
& \left\{(w+2)(11 w+4)[(2-p) w+1]\left(\frac{4 w+2}{w+2}\right)^{n}\right. \\
& \left.+2(2 w+1)\left[w(11 w+4) p+5 w^{2}+8 w-4\right]\right\} .
\end{aligned}
$$

Substituting equations (24), (25) and (50) into equation (46) yields

$$
P_{n, \text { tot }}^{(n)}=\frac{3^{n-2}}{4 p+6}\left[(9-4 p)\left(2 \times 3^{n}+n\right)+4(9+p)\right]
$$

and

$$
\begin{aligned}
P_{n, \text { tot }}^{(n)}= & \frac{1}{54 w[(p+1) w+2]} \\
& \left\{\frac{3[(2-p) w+1](11 w+4)(w+2)}{2(2 w+1)}\left(\frac{12 w+6}{w+2}\right)^{n}\right. \\
& +\frac{27 w[(2-p) w+1](5 w-8)(w+2)}{(2 w-8)(2 w+1)}\left(\frac{4 w+2}{w+2}\right)^{n} \\
& +\frac{3^{n}}{w-4}\left[6(7 p-5) w^{3}-12(4 p+9) w^{2}\right. \\
& -24(2 p+3) w+48]\},
\end{aligned}
$$

for $w=4$ and $w \neq 4$, respectively.

Then, according to equation (45), the explicit expression for ATT is

$$
\langle P\rangle_{n}=\frac{2 \times 3^{n-2}}{(4 p+6)\left(3^{n}+1\right)}\left[(9-4 p)\left(2 \times 3^{n}+n\right)+4(9+p)\right]
$$

and

$$
\begin{aligned}
\langle P\rangle_{n}= & \frac{2}{54 w[(p+1) w+2]\left(3^{n}+1\right)} \\
& \left\{\frac{3[(2-p) w+1](11 w+4)(w+2)}{2(2 w+1)}\left(\frac{12 w+6}{w+2}\right)^{n}\right. \\
& +\frac{27 w[(2-p) w+1](5 w-8)(w+2)}{(2 w-8)(2 w+1)}\left(\frac{4 w+2}{w+2}\right)^{n} \\
& +\frac{3^{n}}{w-4}\left[6(7 p-5) w^{3}-12(4 p+9) w^{2}\right. \\
& -24(2 p+3) w+48]\},
\end{aligned}
$$

for $w=4$ and $w \neq 4$, respectively.

For the particular case $w=1$, equation (54) reduces to equation (40); and for the special case $p=0$, equations (53) and (54) reduce to equations (26) and (27), respectively. Thus, for these two limiting cases, equations (53) and (54) are correct. Furthermore, we have also verified the exact expressions given by equations (53) and (54) 
against direct computation from equation (8). For various $w$ and $p$, the analytical and numerical results agree with each other.

Dominating scaling for ATT and its prefactor. For a very large network, according to equations (53) and (54), the leading term of $\langle P\rangle_{n}$ can be expressed in terms of network size $N_{n}$ in a single formula as

$$
\langle P\rangle_{n} \approx \frac{(2-p) w+1}{(1+p) w+2} \frac{(11 w+4)(w+2)}{18 w}\left(2 N_{n}\right)^{\log _{3} \frac{4 w+2}{w+2}} .
$$

From equation (55), we can see that the weight parameter $w$ determines the dominating scaling of $\langle P\rangle_{n}$, and that parameters $w$ and $p$ together determine the prefactor. Therefore, in order to obtain needed trapping efficiency, one can first adjust parameter $w$ to attain the desired dominating scaling, then continue to change parameter $p$ until the prefactor is desired.

Discussion. We have proposed three techniques controlling the efficiency for trapping in a small-world scale-free network with an immobile trap located at an initial node. The first method can qualitatively change the leading scaling of trapping efficiency, which is realized by introducing a parameter governing the weight of each edge. By tuning the weight parameter, the ATT can scale superlinearly, linearly, or sublinearly with the system size. The second approach is based on delayed random walks, controlled by a stochastic parameter that has little influence on the leading scaling of ATT but can significantly affect its prefactor. The third method is a combination of the firth two approaches, it can thus vary both the leading scaling of ATT and its prefactor. For the three trapping problems, we have studied both numerically and analytically the ATT, the results of which are in good agreement with each other. We expect that similar techniques can also be applied to control other dynamics performed in complex networked systems, such as synchronization $^{58}$, navigation ${ }^{59}$, and search $^{60}$.

1. Bar-Haim, A. \& Klafter, J. Geometric versus energetic competition in light harvesting by dendrimers. J. Phys. Chem. B 102, 1662-1664 (1998).

2. Bar-Haim, A. \& Klafter, J. Dendrimers as light harvesting antennae. J. Lumin. 76-77, 197-200 (1998)

3. Bar-Haim, A., Klafter, J. \& Kopelman, R. Dendrimers as controlled artificial energy antennae. J. Am. Chem. Soc. 119, 6197-6198 (1997).

4. Bentz, J. L., Hosseini, F. N. \& Kozak, J. J. Influence of geometry on light harvesting in dendrimeric systems. Chem. Phys. Lett. 370, 319-326 (2003).

5. Bentz, J. L. \& Kozak, J. J. Influence of geometry on light harvesting in dendrimeric systems. II. $n$ th-nearest neighbor effects and the onset of percolation. J. Lumin. 121, 62-74 (2006).

6. Sokolov, I. M., Mai, J. \& Blumen, A. Paradoxal diffusion in chemical space for nearest-neighbor walks over polymer chains. Phys. Rev. Lett. 79, 857-860 (1997).

7. Blumen, A. \& Zumofen, G. Energy transfer as a random walk on regular lattices. J. Chem. Phys. 75, 892-907 (1981).

8. Jasch, F. \& Blumen, A. Target problem on small-world networks. Phys. Rev. E 63, 041108 (2001)

9. Shlesinger, M. F. Mathematical physics: Search research. Nature 443, 281-282 (2006).

10. Montroll, E. W. Random walks on lattices. III. Calculation of first passage times with application to exciton trapping on photosynthetic units. J. Math. Phys. 10, 753-765 (1969).

11. Redner, S. A guide to first-passage processes (Cambridge University Press, Oxford, 2001).

12. Noh, J. D. \& Rieger, H. Random walks on complex networks. Phys. Rev. Lett. 92, 118701 (2004).

13. Condamin, S., Bénichou, O. \& Moreau, M. First-passage times for random walks in bounded domains. Phys. Rev. Lett. 95, 260601 (2005).

14. Condamin, S., Bénichou, O. \& Klafter, J. First-passage time distributions for subdiffusion in confined geometry. Phys. Rev. Lett. 98, 250602 (2007).

15. Condamin, S., Bénichou, O. \& Moreau, M. Random walks and brownian motion: A method of computation for first-passage times and related quantities in confined geometries. Phys. Rev. E 75, 021111 (2007).

16. Condamin, S., Bénichou, O., Tejedor, V., Voituriez, R. \& Klafter, J. First-passage times in complex scale-invariant media. Nature 450, 77-80 (2007).

17. Garza-López, R. A. \& Kozak, J. J. Invariance relations for random walks on squareplanar lattices. Chem. Phys. Lett. 406, 38-43 (2005).
18. Garza-López, R. A., Linares, A., Yoo, A., Evans, G. \& Kozak, J. J. Invariance relations for random walks on simple cubic lattices. Chem. Phys. Lett. 421, 287-294 (2006).

19. Kozak, J. J. \& Balakrishnan, V. Analytic expression for the mean time to absorption for a random walker on the Sierpinski gasket. Phys. Rev. E 65, 021105 (2002).

20. Bentz, J. L., Turner, J. W. \& Kozak, J. J. Analytic expression for the mean time to absorption for a random walker on the Sierpinski gasket. II. The eigenvalue spectrum. Phys. Rev. E 82, 011137 (2010).

21. Wu, B., Lin, Y., Zhang, Z. Z. \& Chen, G. R. Trapping in dendrimers and regular hyperbranched polymers. J. Chem. Phys. 137, 044903 (2012).

22. Lin, Y. \& Zhang, Z. Z. Influence of trap location on the efficiency of trapping in dendrimers and regular hyperbranched polymers. J. Chem. Phys. 138, 094905 (2013).

23. Lin, Y., Wu, B. \& Zhang, Z. Z. Determining mean first-passage time on a class of treelike regular fractals. Phys. Rev. E 82, 031140 (2010).

24. Zhang, Z. Z., Guan, J. H., Xie, W. L., Qi, Y. \& Zhou, S. G. Random walks on the Apollonian network with a single trap. EPL 86, 10006 (2009).

25. Zhang, Z. Z., Yang, Y. H. \& Gao, S. Y. Role of fractal dimension in random walks on scale-free networks. Eur. Phys. J. B 84, 331-338 (2011).

26. Zhang, Z. Z., Xie, W. L., Zhou, S. G., Li, M. \& Guan, J. H. Distinct scalings for mean first-passage time of random walks on scale-free networks with the same degree sequence. Phys. Rev. E 80, 061111 (2009).

27. Lin, Y., Julaiti, A. \& Zhang, Z. Z. Mean first-passage time for random walks in general graphs with a deep trap. J. Chem. Phys. 137, 124104 (2012).

28. Lin, Y. \& Zhang, Z. Z. Mean first-passage time for maximal-entropy random walks in complex networks. Sci. Rep. 4, 5365 (2014).

29. Peng, X. \& Zhang, Z. Z. Maximal entropy random walk improves efficiency of trapping in dendrimers. J. Chem. Phys. 140, 234104 (2014).

30. Liu, Y.-Y., Slotine, J.-J. \& Barabási, A.-L. Controllability of complex networks Nature 473, 167-173 (2011).

31. Yuan, Z. Z., Zhao, C., Di, Z. R., Wang, W.-X. \& Lai, Y.-C. Exact controllability of complex networks. Nat. Commun. 4, 2447 (2013).

32. Liu, Y.-Y., Slotine, J.-J. \& Barabási, A.-L. Observability of complex systems. Proc. Natl. Acad. Sci. 110, 2460-2465 (2013).

33. Wang, W.-X., Ni, X., Lai, Y.-C. \& Grebogi, C. Optimizing controllability of complex networks by minimum structural perturbations. Phys. Rev. E 85, 026115 (2012).

34. Yan, G., Ren, J., Lai, Y.-C., Lai, C.-H. \& Li, B. W. Controlling complex networks: How much energy is needed? Phys. Rev. Lett. 108, 218703 (2012).

35. Liu, Y.-Y., Slotine, J.-J. \& Barabási, A.-L. Control centrality and hierarchical structure in complex networks. PLoS ONE 7, e44459 (2012).

36. Nepusz, T. \& Vicsek, T. Controlling edge dynamics in complex networks. Nat. Phys. 8, 568-573 (2012).

37. Jia, T. et al. Emergence of bimodality in controlling complex networks. Nat. Commun. 4, 2002 (2013).

38. Jia, T. \& Barabási, A.-L. Control capacity and a random sampling method in exploring controllability of complex networks. Sci. Rep. 3, 2354 (2013).

39. Bar-Haim, A. \& Klafter, J. On mean residence and first passage times in finite onedimensional systems. J. Chem. Phys. 109, 5187-5193 (1998).

40. Wu, B. \& Zhang, Z. Z. Controlling the efficiency of trapping in treelike fractals. J. Chem. Phys. 139, 024106 (2013).

41. Albert, R. \& Barabási, A.-L. Statistical mechanics of complex networks. Rev. Mod. Phys. 74, 47-97 (2002).

42. Newman, M. E. J. The structure and function of complex networks. SIAM Rev. 45, 167-256 (2003)

43. Newman, M. E. J. Networks: an introduction (Cambridge University Press, Oxford, 2010).

44. Boettcher, S., Singh, V. \& Ziff, R. M. Ordinary percolation with discontinuous transitions. Nat. Commun. 3, 787 (2012).

45. Dorogovtsev, S. N., Goltsev, A. V. \& Mendes, J. F. F. Pseudofractal scale-free web Phys. Rev. E 65, 066122 (2002).

46. Bollt, E. M. \& ben-Avraham, D. What is special about diffusion on scale-free nets? New J. Phys. 7, 26 (2005).

47. Rozenfeld, H. D., Havlin, S. \& ben-Avraham, D. Fractal and transfractal recursive scale-free nets. New J. Phys. 9, 175 (2007).

48. Rozenfeld, H. D. \& Ben-Avraham, D. Percolation in hierarchical scale-free nets. Phys. Rev. E 75, 061102 (2007).

49. Zhang, Z. Z., Zhou, S. G. \& Chen, L. C. Evolving pseudofractal networks. Eur. Phys. J. B 58, 337-344 (2007).

50. Zhang, Z. Z., Liu, H. X., Wu, B. \& Zhou, S. G. Enumeration of spanning trees in a pseudofractal scale-free web. EPL 90, 68002 (2010).

51. Hasegawa, T. \& Nemoto, K. Hierarchical scale-free network is fragile against random failure. Phys. Rev. E 88, 062807 (2013).

52. Friesecke, G. Convergence to equilibrium for delay-diffusion equations with small delay. J. Dyn. Diff. Equat. 5, 89-103 (1993).

53. Hale, J. \& Lunel, S. V. Effects of small delays on stability and control (Birkhäuser Basel, 2001).

54. Watts, D. J. \& Strogatz, S. H. Collective dynamics of small-worldnetworks. Nature 393, 440-442 (1998).

55. Barrat, A., Barthélemy, M., Pastor-Satorras, R. \& Vespignani, A. The architecture of complex weighted networks. Proc. Natl. Acad. Sci. 101, 3747-3752 (2004). 
56. Lin, Y. \& Zhang, Z. Z. Random walks in weighted networks with a perfect trap: An application of Laplacian spectra. Phys. Rev. E 87, 062140 (2013).

57. Zhang, Z. Z., Qi, Y., Zhou, S. G., Xie, W. L. \& Guan, J. H. Exact solution for mean first-passage time on a pseudofractal scale-free web. Phys. Rev. E 79, 021127 (2009).

58. Barahona, M. \& Pecora, L. M. Synchronization in small-world systems. Phys. Rev. Lett. 89, 054101 (2002).

59. Kleinberg, J. M. Navigation in a small world. Nature 406, 845-845 (2000).

60. Bénichou, O., Loverdo, C., Moreau, M. \& Voituriez, R. Intermittent search strategies. Rev. Mod. Phys. 83, 81-129 (2011).

\section{Acknowledgments}

The authors thank Yihang Yang for his assistance in preparing this manuscript. This work was supported by the National Natural Science Foundation of China under Grants No. 11275049 and the National Basic Research Program of China under Grant No. 2010CB731401.

\section{Author contributions}

Y.L. and Z.Z.Z. designed the research, performed the research, and wrote the manuscript.

\section{Additional information}

Competing financial interests: The authors declare no competing financial interests.

How to cite this article: Lin, Y. \& Zhang, Z. Controlling the efficiency of trapping in a scale-free small-world network. Sci. Rep. 4, 6274; DOI:10.1038/srep06274 (2014).

This work is licensed under a Creative Commons Attribution-NonCommercialNoDerivs 4.0 International License. The images or other third party material in this article are included in the article's Creative Commons license, unless indicated otherwise in the credit line; if the material is not included under the Creative Commons license, users will need to obtain permission from the license holder in order to reproduce the material. To view a copy of this license, visit http:// creativecommons.org/licenses/by-nc-nd/4.0/ 